\title{
Future directions in Agile research: Alignment and divergence between
} research and practice: Agile Product Development Special Issue Of J. Softw. Evol. And Proc.
Article in Journal of Software: Evolution and Process · June 2017 DOI: $10.1002 / \mathrm{smr} .1884$
5 authors, including:

Some of the authors of this publication are also working on these related projects:

FLEXI project View project

software architecture analysis of usability View project 


\title{
Future Directions in Agile Research: Alignments and Divergence between Research and Practice
}

\author{
Alok Mishra $^{1}$, Juan Garbajosa ${ }^{2}$, Xiaofeng Wang ${ }^{3}$, Jan Bosch $^{4}$ and Pekka Abrahamsson ${ }^{5}$ \\ ${ }^{1}$ Atilim University, Ankara, Turkey \\ ${ }^{2}$ Technical University of Madrid, Madrid, Spain \\ ${ }^{3}$ University of Bozen-Bolzano, Bolzano, Italy \\ ${ }^{4}$ Chalmers University of Technology, Charlmers, Sweden \\ ${ }^{5}$ Norwegian University of Science and Technology, Trondheim, Norway
}

Received ...

\section{INTRODUCTION}

Since the publication of Agile Manifesto in 2001, agile methods have transited from a grassroot initiative among enthusiastic advocates and developers to a mainstream software development approach adopted by both small and large companies worldwide [1]. Meanwhile research on agile methods has grown rapidly and steadily into an established research area, evidenced by dedicated conferences (e.g., XP conference series, research track of previous Agile Conference series), special issues and sections in top Information Systems and Software Engineering journals (e.g. [2, 3, 4]). However, practitioners and consultants have largely driven the advancement in agile field, and agile research has lagged behind practice in the past [2]. Has this situation changed as both agile methods and research community become increasingly mature? To be able to answer such questions, there is a constant need to check what interest agile practitioners and what agile researchers are investigating, to make sure that the states of the art and practice are aligned properly.

\section{THE STATE OF THE PRACTICE}

Agile conference is an annual conference dedicated to "furthering Agile principles and providing a venue for people and ideas to flourish", where "the Agile tribes meet." It is supported by the Agile Alliance (www.agilealliance.org) and mainly run by agile practitioners. The talks and workshops are grouped under so-called "stages". Each "stage" reflects a significant topic that interests agile practitioners. We carefully reviewed the stages that were run at the Agile conference from 2011 to 2016. The reason why we chose this period was because that the information on each year's program was available only for these years, not before 2011, at the time this article was written. Table I shows what topics are covered at the recent Agile Conferences and their continuity over the last six years.

It is worth pointing out that the first group of topics are constantly present at the conference and occupying the attention of the practitioners. The second group represents the emerging topics. It is not surprising to see that DevOps is one of them. In contrast, Adoption and transformation has not been a main topic in the past four editions of the conference. This may be seen as a sign that agile methods have passed early innovation adoption phase and become mainstream as demonstrated in the 10th annual state of agile report from VersionOne [1]. Therefore adoption and transformation, apparently, is no more a central concern for practitioners. The last group of topics 


\begin{tabular}{lcccccc}
\hline Stages & 2011 & 2012 & 2013 & 2014 & 2015 & 2016 \\
\hline Business value and working with cus- & $\checkmark$ & $\checkmark$ & $\checkmark$ & $\checkmark$ & $\checkmark$ & $\checkmark$ \\
tomers/stakeholders & & & & & & \\
Coaching and mentoring & $\checkmark$ & $\checkmark$ & $\checkmark$ & $\checkmark$ & $\checkmark$ & $\checkmark$ \\
Collaboration culture and Teams & $\checkmark$ & $\checkmark$ & $\checkmark$ & $\checkmark$ & $\checkmark$ & $\checkmark$ \\
Development languages, practices, tech- & $\checkmark$ & $\checkmark$ & $\checkmark$ & $\checkmark$ & $\checkmark$ & $\checkmark$ \\
niques and craftsmanship & & & & & & \\
Enterprise agile & $\checkmark$ & $\checkmark$ & $\checkmark$ & $\checkmark$ & $\checkmark$ & $\checkmark$ \\
Leadership & $\checkmark$ & $\checkmark$ & $\checkmark$ & $\checkmark$ & $\checkmark$ & $\checkmark$ \\
Testing and quality assurance & $\checkmark$ & $\checkmark$ & $\checkmark$ & $\checkmark$ & $\checkmark$ & $\checkmark$ \\
User experience and Interaction design & $\checkmark$ & $\checkmark$ & $\checkmark$ & $\checkmark$ & $\checkmark$ & $\checkmark$ \\
Business and project and portfolio man- & $\checkmark$ & & $\checkmark$ & $\checkmark$ & $\checkmark$ & $\checkmark$ \\
agement & & & & & & \\
\hline DevOps & & & $\checkmark$ & $\checkmark$ & $\checkmark$ & $\checkmark$ \\
Learning & & & & & $\checkmark$ & $\checkmark$ \\
Government & $\checkmark$ & $\checkmark$ & & & & \\
\hline Adoption and Transformation & $\checkmark$ & & & & & \\
\hline Agile for embedded systems development & $\checkmark$ & & & & & \\
Distributed agile & & & $\checkmark$ & & & \\
Crowdsourced & & & $\checkmark$ & & & \\
Lean Startup & & & \\
\hline
\end{tabular}

Table I. The stages run at the Agile Conferences

only made their appearance once in the conference in the past six years. It is somehow surprising to see that Crowdsourced and Lean Startup have not been continuously the main topics.

\section{ALIGNMENT AND DIVERGENCE OF AGILE RESEARCH WITH PRACTICE}

After the review of the topics occupying the attention of agile practitioners, the natural questions to ask are: what agile researches are studying, and are the practice and research aligned? If not, what are the gaps? To shed light on these questions, we turned to the primary research-oriented conference dedicated to agile software development - XP Conference series, which over the years have transformed from a mainly European conference to a truly international forum for agile researchers worldwide. We surveyed the research papers published at the conference in the past six years, based on their titles and keywords, and mapped them to the Agile stages listed in Table I. The mapping result is shown in Table II.

Comparing Table I with Table II, we can see that the constant topics that practitioners are interested in, including Business value and working with customers/stakeholders, Collaboration culture and teams, Testing and quality assurance and Business and project and portfolio management, also received continuous scrutinization by research. Similarly Development languages, practices, techniques and craftsmanship is also a constant research topic, even though to a less extent relatively. Enterprise agile, i.e., scaling agile from the team to organization level, has been the focus of quite a few research papers in the past four years. It is worth noting that, even though the research on User experience and interaction design also shows continuity, before 2014 the keywords used in the research papers were mainly graphical user interface and usability. User experience has appeared as a keyword only since 2014. In comparison it has been used as the stage name in Agile Conference already in 2011, if not earlier.

Noticeably two constant topics at Agile Conference apparently received very little attention by the research community in terms of the focused research papers published at XP: Leadership and Coaching and mentoring. This gap indicates that more research effort may be needed to meet the interests of practitioners. On the contrary, Adoption and Transformation is a popular topic that researchers publish on but receives diminishing attention from the practical world. However it is worth noting that Leadership and Coaching and mentoring are regarded as two issues fundamental 


\begin{tabular}{lcccccc}
\hline Stages & 2011 & 2012 & 2013 & 2014 & 2015 & 2016 \\
\hline $\begin{array}{l}\text { Business value and working with cus- } \\
\text { tomers/stakeholders }\end{array}$ & $\checkmark$ & $\checkmark$ & $\checkmark$ & $\checkmark$ & $\checkmark$ & $\checkmark$ \\
$\begin{array}{l}\text { Coaching and mentoring } \\
\text { Collaboration culture and Teams }\end{array}$ & & & $\checkmark$ & & & \\
$\begin{array}{l}\text { Development languages, practices, tech- } \\
\text { niques and craftsmanship }\end{array}$ & $\checkmark$ & & $\checkmark$ & $\checkmark$ & & $\checkmark$ \\
Enterprise agile & & & $\checkmark$ & $\checkmark$ & $\checkmark$ & $\checkmark$ \\
$\begin{array}{l}\text { Leadership } \\
\text { Testing and quality assurance }\end{array}$ & $\checkmark$ & & & & & \\
User experience and Interaction design & $\checkmark$ & $\checkmark$ & $\checkmark$ & $\checkmark$ & $\checkmark$ & $\checkmark$ \\
$\begin{array}{l}\text { Business and project and portfolio man- } \\
\text { agement }\end{array}$ & $\checkmark$ & $\checkmark$ & $\checkmark$ & $\checkmark$ & $\checkmark$ & $\checkmark$ \\
\hline $\begin{array}{l}\text { DevOps } \\
\text { Learning }\end{array}$ & & $\checkmark$ & $\checkmark$ & $\checkmark$ & & $\checkmark$ \\
Government & & & & & & \\
\hline Adoption and Transformation & $\checkmark$ & $\checkmark$ & $\checkmark$ & $\checkmark$ & $\checkmark$ & $\checkmark$ \\
\hline $\begin{array}{l}\text { Agile for embedded systems development } \\
\text { Distributed agile }\end{array}$ & $\checkmark$ & $\checkmark$ & $\checkmark$ & & $\checkmark$ & \\
$\begin{array}{l}\text { Crowdsourced } \\
\text { Lean Startup }\end{array}$ & & & & & & $\checkmark$ \\
\hline
\end{tabular}

Table II. The Mapping of research papers published at the XP conference to the Agile Conference stages

for transformation [5, 6]. Therefore there is a need to validate the claimed gap and trend through analysing in depth the published papers at XP and the talks given at Agile conferences.

In line with the industry interest, DevOps also became a main research topic at XP Conference in the last couple of years, even though two years later after Agile Conference. A similar pattern can be observed with the topic of Lean Startup. Instead, Agile for embedded systems development and Distributed agile are recurring research topics in contrast to its once-off occurrence at Agile Conference. Two topics that have been covered by practitioners, Government and Crowdsourced, did not make to the list of keywords in the research papers surveyed.

Finally, several key topics that researchers keep publishing on, are not covered by the stages of Agile Conference, including software measurement, visualization, maintenance and process simulation in the agile context. Similarly to what has been suggested before, it would be worthwhile to research the roots behind this apparent divergence, such as the composition of the communities that attend the conferences, lack of maturity of research results, or lack of connection of research communities to tool makers. Finding answers to this divergence may provide clues on how to improve both align research and practice.

\section{CONTRIBUTION OF THE SPECIAL ISSUE PAPERS}

Using Tables I and II as the backdrop, we could better position the five papers accepted in this special issue, and understand the contribution they made to the body of knowledge of agile research.

Project, program and portfolio management has always been a core topic that interests both agile practitioners and researchers alike. Two papers in this special issue fit broadly to this branch. In the paper titled "Assisting the continuous improvement of Scrum projects using metrics and Bayesian networks", Mirko Perkusich, Kyller Costa Gorgonio, Hyggo Almeida and Angelo Perkusich apply the technique of Bayesian networks to assist the continuous improvement of Scrum projects. The built Bayesian network provides data to help Scrum Masters lead the improvement of business value delivery of Scrum teams. The authors have validated the Bayesian network inferences with 10 simulated scenarios. Based on the improved accuracy of the inferences, the authors believe that the Bayesian networks adequately represent Scrum projects from the perspective of a Scrum master and the model built can be adapted to any Scrum team. 
Instead, the other paper "An empirical study of portfolio management and Kanban in agile and lean software companies" focuses on the level of portfolio management. To understand whether traditional portfolio management tools and methods are applicable for software companies adopting agile and lean approaches due to the complex preplanning activities required, the authors, Muhammad Ovais Ahmad, Lucy Ellen Lwakatare, Pasi Kuvaja and Markku Oivo have reviewed traditional portfolio management tools and methods that have been conventionally used in industry and presented in literature. Especially their use in agile and lean software companies have been investigated using interviews in seven agile and lean software companies in Finland. Similar to what happens to the adoption of any method or tool, the findings of the study indicate that agile and lean companies do not use traditional portfolio management tools and methods in their entirety and adapt suitable components from them into their own practices. In addition, new portfolio management tools and methods are invented, to cope with the dynamics required by agile and lean methods. The study reveals that what matters the most to agile and lean companies is to facilitate immediate feedback from customers to assess the value of offerings in their portfolios. A growing interest is also detected in using Kanban at the portfolio level to provide a visual overview of offerings.

"Cluster-based test cases prioritization and selection technique for agile regression testing", authored by Passant Kandil, Sherin Moussa and Nagwa Badr, follows the continuous industry and research interests on Testing and quality assurance. It investigates specifically regression testing. As the authors claim, regression testing has seen a remarkable progress with the increasing popularity of agile methods in recent years, which stresses the central role of regression testing in maintaining software quality. In this paper, the authors present an automated agile regression testing approach at both the sprint and release levels, which addresses both Weighted Sprint Test cases Prioritization (WSTP) technique and Cluster-based Release Test cases Selection (CRTS) technique. The proposed approach has been evaluated through two experiments using three different datasets representing three different system releases, and achieves enhancement for both prioritization and selection of test cases for agile regression testing. The significant contribution of this research is the usage of agile parameters for regression testing of agile projects specifically.

Another ongoing topic that has high relevance for practice and sees the increasing popularity in agile research, Enterprise agile (i.e., scaling agile), has been further examined in the paper "Assessing the adoption level of scaled agile development: a maturity model for Scaled Agile Framework" from Oktay Turetken, Igor Stojanov and Jos J. M. Trienekens. The study is focused on Scaled Agile Framework (SAFe), which has emerged as a popular agile scaling framework much discussed in the agile community. However, as the authors state, there is a lack of a well-structured gradual approach for establishing SAFe, and organizations can benefit greatly from a uniform model for assessing the current progress and establishing a roadmap for the initiative before and during SAFe adoption. In this study, the authors developed a maturity model by extending an existing agile maturity model with the key SAFe practices. The work has been undertaken in close collaboration with industry experts using the Delphi technique, and the model has been evaluated in a large company. It can provide guidance for software developing organizations to build SAFe adoption roadmaps as well as to assess the levels of their SAFe adoption.

DevOps has been a significant and popular topic emerged most recently in both practice and research fields, as shown in the previous sections. "A Qualitative Study of DevOps Usage in Practice", authored by Floris Erich, Chintan Amrit and Maya Daneva, contributes to the growing understanding of this phenomenon. The authors observe that little empirical research has been performed to study the ways in which organizations implement DevOps and the outcomes they obtain. The objective of the study therefore is to offer a better understanding of both the state of the art and the state of the practice of DevOps implementation. A Systematic Literature Review on what empirical researchers have written has been conducted first to depict the state of the art. Then interviews in six organizations of various sizes and in various industries have been undertaken to draw the state of the practice. The study discovers that the literature and the interviewed organizations define DevOps in different ways, but a common theme exists, which is the need for interaction between development and operation, which can take place between individuals, teams 
and departments using various principles and practices. The main contribution of the study is a conceptual framework that researchers and practitioners can use to further study DevOps.

In summary, the papers included in this special issue have addressed the major current concerns of the industry, meanwhile also capturing the emerging new trends that are significant to both practice and research. Thus this special issue can be seen as a collective effort of agile researchers to align research effort with the state of the practice.

\section{ACKNOWLEDGEMENT}

We would like to thank all the reviewers who contributed to this special issue with their thoughtful and constructive comments that helped the authors improve their papers.

\section{REFERENCES}

1. VersionOne. 2016. The 10th Annual State of Agile Report. URL

2. Abrahamsson P, Conboy K, Wang X. 2009. "Lots done, more to do": the current state of agile systems development research. European Journal of Information Systems. Vol.18, 281-284.

3. Dingsyr T, Nerur S, Balijepally VG, Moe NB. 2012. A decade of agile methodologies: Towards explaining agile software development. Journal of Systems and Software. Vol. 85(6), 1213-1221.

4. Dingsyr T, Lassenius C. 2016. Emerging themes in agile software development: Introduction to the special section on continuous value delivery. Information and Software Technology. Vol.77, 56-60.

5. Parry K W. 1998. Grounded theory and social process: A new direction for leadership research. The Leadership Quarterly. Vol.9, Issue 1, 85-105, ISSN 1048-9843.

6. Gandomani T J, Zulzalil H, Ghani A, Sultan A, Parizi R. The impact of inadequate and dysfunctional training on Agile transformation process: A Grounded Theory study. Information and Software Technology. Vol.57, 295-309. 